\title{
A new lot sizing and scheduling heuristic for multi-site biopharmaceutical production
}

\author{
Folarin B. Oyebolu ${ }^{1}$ • Jeroen van Lidth de Jeude ${ }^{2}$ • \\ Cyrus Siganporia ${ }^{3}$. Suzanne S. Farid ${ }^{3}$. \\ Richard Allmendinger ${ }^{4} \cdot$ Juergen Branke $^{1}$ B
}

Received: 23 May 2016 / Revised: 1 March 2017 / Accepted: 9 May 2017 / Published online: 29 May 2017 (C) The Author(s) 2017. This article is an open access publication

\begin{abstract}
Biopharmaceutical manufacturing requires high investments and longterm production planning. For large biopharmaceutical companies, planning typically involves multiple products and several production facilities. Production is usually done in batches with a substantial set-up cost and time for switching between products. The goal is to satisfy demand while minimising manufacturing, set-up and inventory costs. The resulting production planning problem is thus a variant of the capacitated lotsizing and scheduling problem, and a complex combinatorial optimisation problem. Inspired by genetic algorithm approaches to job shop scheduling, this paper proposes a tailored construction heuristic that schedules demands of multiple products sequentially across several facilities to build a multi-year production plan (solution). The
\end{abstract}

\footnotetext{
$凶 \quad$ Juergen Branke

juergen.branke@wbs.ac.uk

Folarin B. Oyebolu

f.b.oyebolu@warwick.ac.uk

Jeroen van Lidth de Jeude

j.van-lidth-de-jeude@warwick.ac.uk

Cyrus Siganporia

c.siganporia@ucl.ac.uk

Suzanne S. Farid

s.farid@ucl.ac.uk

Richard Allmendinger

richard.allmendinger@manchester.ac.uk

1 Warwick Business School, University of Warwick, Coventry CV4 7AL, UK

2 Centre for Complexity Science, University of Warwick, Coventry CV4 7AL, UK

3 Department of Biochemical Engineering, University College London, London WC1E 7JE, UK

4 Alliance Manchester Business School, University of Manchester, Manchester M13 9SS, UK
} 
sequence in which the construction heuristic schedules the different demands is optimised by a genetic algorithm. We demonstrate the effectiveness of the approach on a biopharmaceutical lot sizing problem and compare it with a mathematical programming model from the literature. We show that the genetic algorithm can outperform the mathematical programming model for certain scenarios because the discretisation of time in mathematical programming artificially restricts the solution space.

Keywords Evolutionary algorithm $\cdot$ Heuristics $\cdot$ Scheduling · Biopharmaceutical manufacture $\cdot$ Capacity planning $\cdot$ Construction heuristic

\section{Introduction}

The production of biopharmaceuticals is an expensive and time-consuming endeavour. The average cost to bring a new biopharmaceutical to market is estimated at \$1.2-1.8 billion given the high attrition rates (DiMasi and Grabowski 2007; Paul et al. 2010), and building large multiproduct manufacturing facilities can take 4-5 years to complete and costs \$40-650 million (Farid 2007). Given the high cost and long timeframes, biopharmaceutical companies have to plan ahead over a long time horizon, based on a demand forecast for each time period. It is important that production schedules are optimised to make best use of the available production capacity, and even small improvements can have a substantial impact on a company's profit.

Biopharmaceutical production is typically done in a batch-wise manner, with substantial set-up cost and time for switching between products, and a relatively high storage cost. The resulting problem can thus be considered as a variant of the lotsizing and scheduling problem, where a "lot" (or "campaign" as it is often called in this industry) is composed of a set of batches. However, biopharmaceutical production has a number of characteristics that make it challenging to optimise. To spread risk, companies usually have a portfolio of various products, and manufacturing takes place across a network of different facilities, including in-house facilities and outsourced (contract) manufacturing. The facilities' capabilities usually vary with respect to the set of products they can produce, the production rates for different products, batch production costs and batch production times. Furthermore, products have a finite shelf-life and cannot be stored for very long. Overall, biopharmaceutical production constitutes a complex combinatorial optimisation problem.

The current literature on capacity planning in the biopharmaceutical sector is mostly based on mathematical programming models such as, for example, in Lakhdar et al. (2007). Because of the simplifications required to model the problem for mixed integer linear programming, the solution potentially suffers from an artificial restriction of the search space. The goal of this paper is therefore to develop a more flexible metaheuristic approach for the biopharmaceutical lot sizing and scheduling problem, and contrast it with the proposed mixed-integer programming approach as described by Lakhdar et al. (2007).

To this end, we design an genetic algorithm (GA) with an embedded problemspecific construction heuristic. The GA uses an indirect permutation encoding, i.e., the specifically developed construction heuristic schedules demands sequentially in 
the order prescribed by the chromosome. As we demonstrate, the use of an GA allows for a more flexible and realistic model of the real-life problem and avoids some of the simplifications necessitated by available mathematical programming models.

The paper makes three contributions. First, it proposes a new heuristic to solve the multi-site biopharmaceutical lot sizing and scheduling problem that outperforms previously published approaches on a close to real-world case study. Second, it demonstrates that the combination of genetic algorithm and construction heuristic that has been very successful in the scheduling domain can be successfully transferred to tackle complex lot sizing problems. Third, it provides an example for the fact that an exact method based on typical simplifications (in this case fixed time periods) can be worse than a heuristic that does not need to make such simplifications.

The paper is structured as follows. First we provide a brief overview of related work. Section 3 describes in more detail the case study used to evaluate our approach. The GA and the associated construction heuristic are explained in Sect. 4. The results of the empirical evaluation, including a comparison with an MILP approach, are reported in Sect. 5. The paper concludes with a summary and an outlook on future work.

\section{Related work}

Production planning aims to make best use of production resources in order to satisfy production goals or demand over a planning horizon. It is omnipresent in any manufacturing environment. One particular area in production planning is lot sizing and scheduling, which mostly focuses on the trade-off between set-up cost and inventory cost. The basic lot sizing problem was introduced in 1958 by Wagner and Whitin (1958), who considered the case of a single product with deterministic demand. Since then, many different extensions have been considered, reflecting the different environments in different industries. A particularly important extension is to include capacity constraints, resulting in the "capacitated lot sizing problem" (CLSP). Good overviews on the research in this area have been compiled, for example, by Drexl and Kimms (1997), Karimi et al. (2003), and Jans and Degraeve (2008). Recently, Copil et al. (2017) have proposed a classification system for simultaneous lot sizing and scheduling problems.

Very often, CLSPs are modelled as linear or mixed-integer programming problems and solved with software such as IBM's CPLEX (Ramya et al. 2016; Dangelmaier and Kaganova 2013; Walser et al. 1998). However, the CLSP is NP-hard (Bitran and Yanasse 1982), and so there is a limit to the size and complexity of CLSPs that can be tackled with exact mathematical programming methods. For larger and more complex scenarios, various approaches based on meta-heuristics have been proposed. Most of the meta-heuristic approaches use evolutionary algorithms (EAs)-particularly GAs-but also tabu search or particle swarm optimisation have been used, see, e.g., Piperagkas et al. (2012), Jans and Degraeve (2007), and Goren et al. (2008). For a bi-objective CLSP problem, Mehdizadeh et al. (2016) develop two multi-objective meta-heuristic algorithms.

Literature on the capacity planning problem in pharmaceutical or biopharmaceutical industry represent complicated extensions to the CLSP, with multiple products and 
facilities, product-specific manufacturing rates and costs, multi-stage processing, and perishable products. They have applied primarily mathematical programming models based on discrete time-periods which are solved using MILP solver software.

For example, Gatica et al. (2003) and Levis and Papageorgiou (2004) present a mathematical programming approach for the capacity planning problem, but with a focus on long-term planning and capacity investment decisions under clinical trials uncertainty rather than scheduling. Within the context of the biopharmaceutical industry, Lakhdar et al. (2005) developed a mixed-integer linear program for the planning and scheduling of a multi-product biopharmaceutical manufacturing facility and later extended it for use with a multi-facility model where multiple criteria were considered using goal programming (Lakhdar et al. 2007). Siganporia et al. (2014) considered continuous perfusion processes in their planning model as well as variations of bioreactor titres and demand. Each of these models is based on discrete time periods and allows only one product to be manufactured in each time-period. In the case of Lakhdar et al. (2007), where discrete 90 day periods are used, this means that at most four different campaigns (lots) can be scheduled per year and facility. As a result, this effectively artificially restricts the search space.

The GA-based approaches to lot sizing can be broadly divided into approaches using a direct representation or an indirect representation, where the former appears much more often. In a direct representation, the sequence and lot sizes are directly encoded in the chromosome. The main challenge with such an approach is that mutations and crossovers can generate infeasible solutions, which is usually dealt with by discarding those solutions or by special repair operators (Özdamar and Birbil 1998). Methods with an indirect representation use a mapping function/heuristic to derive a production plan from a solution's chromosome. An indirect GA representation has been proposed by Kimms (1999). In his paper, a two-dimensional matrix is used as chromosome, with each entry representing a rule for selecting the set up state for a machine at the end of a period (e.g., the item with maximum holding costs, minimum set up cost, maximum depth, maximum number of predecessors). Thus, this approach can be seen as a selection hyper-heuristic (Burke et al. 2013). To compute the fitness value of a chromosome, a construction scheme is called, which constructs the solution backwards, starting from the end of the planning horizon.

As noted, e.g., by a recent survey (Jans and Degraeve 2008), most meta-heuristics developed for lot sizing are validated only on artificial test data, failing to demonstrate that they can tackle the complexities of real-world problems. Another current research gap is that the vast majority of work on lot sizing assumes that the problems are deterministic, whereas in reality, demand and production rates are usually subject to uncertainty. Integrating uncertainty will require novel approaches, and EAs have already demonstrated some promise in dealing with such problems (Jin and Branke 2005).

A lot more work has been published on GAs for the job-shop scheduling problem (JSP), and they typically use a permutation-based representation, and then apply a construction heuristic to actually construct the schedule based on the permutation (Cheng et al. 1996; Branke and Mattfeld 2005; Bierwirth and Mattfeld 1999). A typical construction heuristic is the Giffler-Thompson algorithm (Giffler and Thompson 1960), which generates active schedules by iteratively selecting the job with the highest pri- 
ority (lowest permutation index) from the set of eligible jobs, and then scheduling it at the earliest possible time. However, this approach cannot be directly transferred to our lot sizing problem, because (i) scheduling as early as possible would lead to excessive storage costs and (ii) the existence of a heterogeneous set of alternative facilities.

Variations of construction heuristics have been used for various types of lot sizing problems. For example, Ho et al. (2006) developed two construction heuristics for the uncapacitated dynamic lot-sizing problem that are extensions of earlier heuristics by Silver and Meal (1973), and show that they outperform six other construction heuristics including the original Silver and Meal heuristic. James and Almada-Lobo (2011) propose, along with other heuristics, a MILP-based 'relax-and-fix' construction heuristic for the parallel-machine capacitated lotsizing and scheduling problem with sequence-dependent setups (CLSD-PM). This construction heuristic solves a sequence of decomposed 'subMILPs' in order to construct an initial solution for the various search algorithms it is coupled with. Ant Colony Optimisation (ACO) has also been used for uncapacitated and capacitated multi-level problems (Pitakaso et al. 2007; Almeder 2010). In both cases, ACO was used to determine production decisions from top items to raw materials and a MILP solver is used to calculate the corresponding production and inventory levels. Finally, Almada-Lobo et al. (2007) propose a five step heuristic for finding good feasible solutions. Each step of the heuristic is either a forward or backward pass (or a combination of both) through the schedule. Further work uses this heuristic as an initial starting solution for meta-heuristic searches (Almada-Lobo and James 2010).

It is interesting to note that the construction heuristics mentioned above operate sequentially in either a forwards or backwards pass through the schedule, or a combination thereof. Instead, the construction heuristic proposed here inserts jobs in an order of importance determined by the GA and not necessarily in any chronological order.

The construction heuristic we propose in this paper is therefore tailored to our problem, and still allows us to use the permutation-based approach that is successful in the job shop scheduling domain.

\section{Industrial case study}

To evaluate our proposed method, we use the biopharmaceutical industrial case study presented by Lakhdar et al. (2007). This is anonymized real world data comprising anticipated market demand and manufacturing facility characteristics. This benchmark problem features multiple products to be produced on multiple facilities with different efficiencies and costs, setup times, batch production, perishable inventory, and the possibility to backlog demand.

The demand forecast comprises a time horizon of 15 years and 15 products ( $\mathrm{p} 1-$ p15). The forecast indicates yearly market demands, assumed to be fulfilled at the end of each year (Table 1$)^{1}$. The demand can be scheduled across 10 facilities (i1-i10), but

\footnotetext{
1 Note that the product 1 demand for year 10 in Table 1 in Lakhdar et al. (2007) was 63, which is not consistent with the general trend of the other years, so we changed it to 163 .
} 


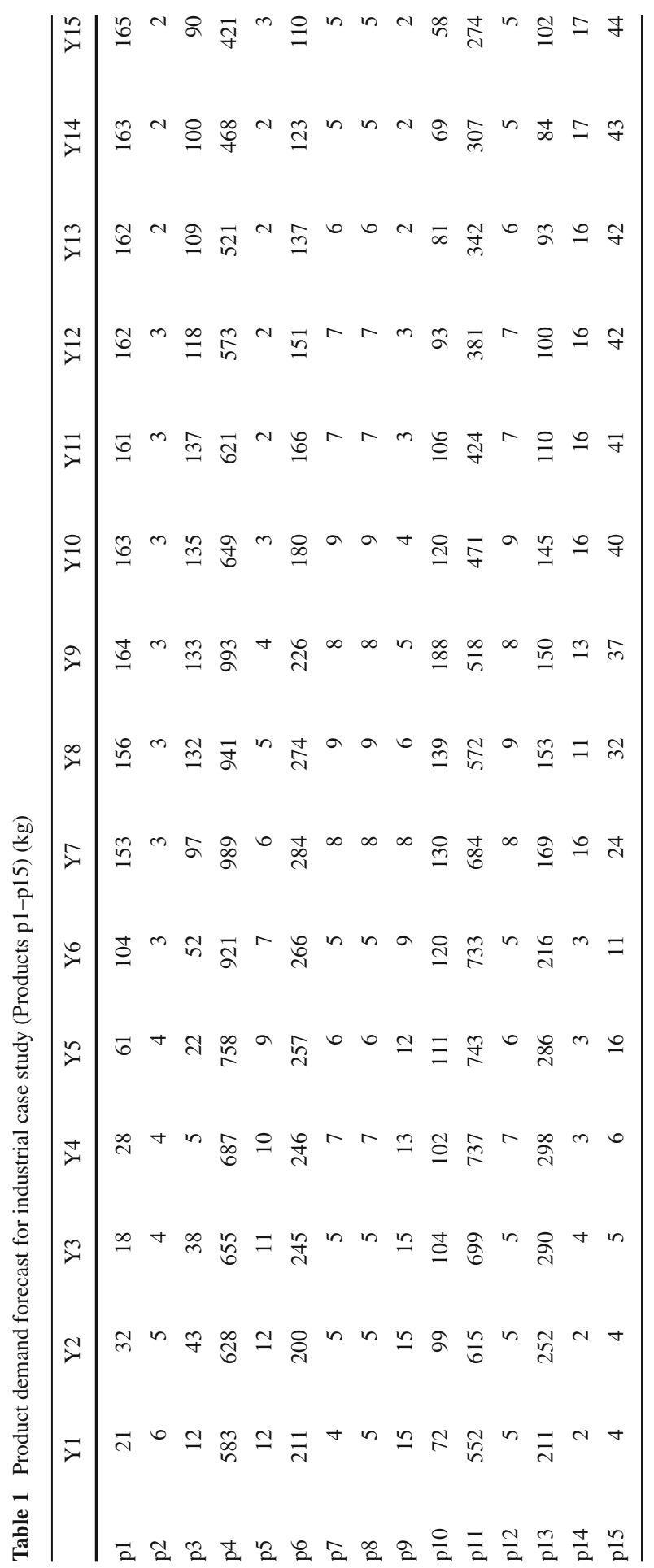


not all facilities can produce all 15 products. All facilities are assumed to be available for the entire time horizon apart from facility 6 (i6) which is unavailable until Y2, and facility 9 (i9) which is unavailable until Y11. Of the ten manufacturing facilities, i1, i4, i6, and i9 are owned facilities while the rest are owned by contract manufacturing organisations (CMO). Production rates (Table 2), manufacturing yields (Table 3 ) and manufacturing costs (Table 4) are specified for all facility-product combinations (RMU in the tables denotes relative monetary unit). The manufacturing yield determines how many kilograms of a specific product are produced in a batch for a specific facility. The manufacturing cost of a product is thus also dependent on the yield. Setup cost and time are incurred when a facility is switching between products. For consecutive batches of the same product, no setup time/cost is involved. There is the additional requirement for setup if the facility has been idle for more than 90 days. This accounts for the extra equipment preparation activities (cleaning, sterilisation, etc.) required after prolonged idle time. There is also a restriction on the time a product may be stored before it has to be thrown away, the so-called maximum shelf-life. In the case that the demand cannot be fulfilled in time, it is backlogged, but there is a backlog penalty for every unit that is not delivered on time. Also, backlogged demand decays exponentially at a rate of $50 \%$ every three months. For example, if a demand of 100 $\mathrm{kg}$ cannot be delivered on time, 6 months later, only $25 \mathrm{~kg}$ could actually be sold, and $75 \mathrm{~kg}$ of the demand would have been lost, reducing the revenue correspondingly.

The case study assumes a fixed sales price, changeover cost, storage cost, and setup time for all products (Table 5). ${ }^{2}$ The setup time includes the time of production of the first batch. In addition, it is assumed that a month is 30 days and, subsequently, a year is equal to 360 days.

The objective is to maximize the overall profit, calculated as total revenue minus the cost for production, storage, setups and backlog penalties. Given a set of heterogeneous facilities with different manufacturing yields, manufacturing cost, and batch production rates for different products, this takes into account maximizing the amount of products sold, and minimizing the manufacturing cost, the storage cost, the setup cost, and any backlog penalty.

Lakhdar et al. (2007) used mixed integer linear programming (MILP) to solve this problem, and a full description of the mathematical programme formulation can be found in the "Appendix". The GA proposed in this paper is subject to the same constraints, except that we do not restrict production on a particular facility to one product per time period. This seemed to be an artificial restriction imposed only to reduce the modelling complexity of the MILP.

\section{Proposed genetic algorithm with construction heuristic}

For job shop scheduling, many successful GAs use indirect encodings, with the GA only searching the space of permutations of jobs. For evaluation, a schedule is con-

\footnotetext{
2 Note that the description by Lakhdar et al. (2007) had some inconsistencies in the units specified, so in Table 5 we updated the units for setup cost, sales price, storage cost and backlog penalty to be consistent with the other data.
} 


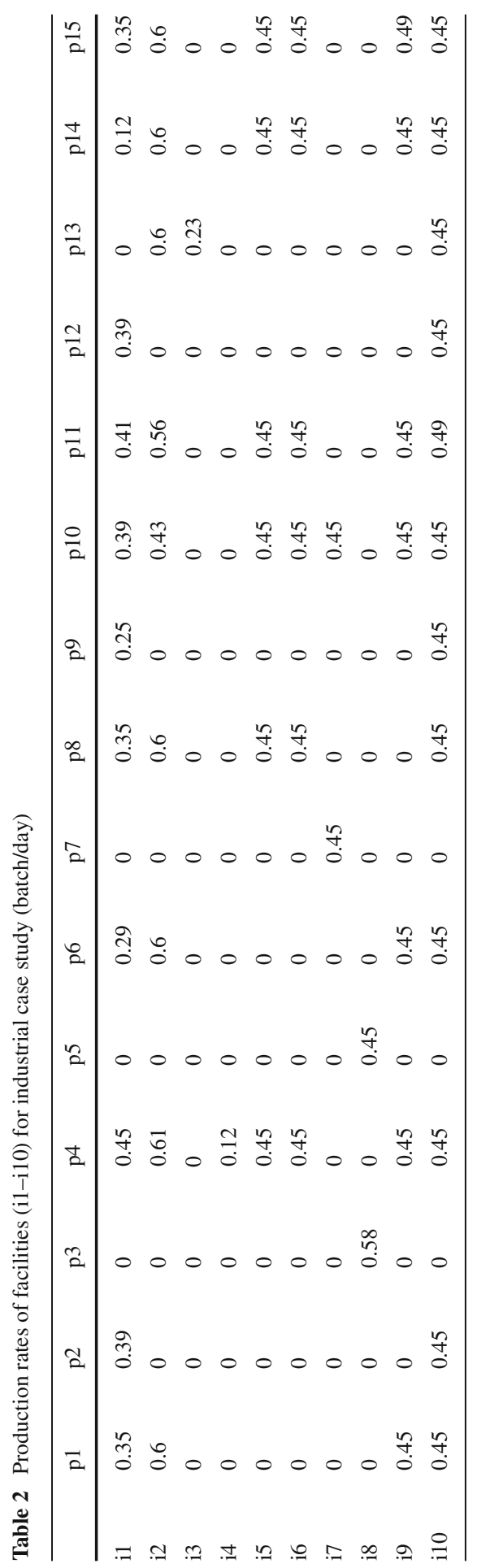


Table 3 Manufacturing yields of facilities for industrial case study (kg/batch)

\begin{tabular}{rrrrrrrrrrrrrrrr}
\hline & $\mathrm{p} 1$ & $\mathrm{p} 2$ & $\mathrm{p} 3$ & $\mathrm{p} 4$ & $\mathrm{p} 5$ & $\mathrm{p} 6$ & $\mathrm{p} 7$ & $\mathrm{p} 8$ & $\mathrm{p} 9$ & $\mathrm{p} 10$ & $\mathrm{p} 11$ & $\mathrm{p} 12$ & $\mathrm{p} 13$ & $\mathrm{p} 14$ & $\mathrm{p} 15$ \\
\hline $\mathrm{i} 1$ & 10 & 1 & 0 & 8 & 0 & 6 & 0 & 10 & 2 & 9 & 7 & 1 & 0 & 12 & 12 \\
$\mathrm{i} 2$ & 9 & 0 & 0 & 8 & 0 & 6 & 0 & 9 & 0 & 8 & 10 & 0 & 10 & 12 & 11 \\
$\mathrm{i} 3$ & 0 & 0 & 0 & 0 & 0 & 0 & 0 & 0 & 0 & 0 & 0 & 0 & 9 & 0 & 0 \\
$\mathrm{i} 4$ & 0 & 0 & 0 & 9 & 0 & 0 & 0 & 0 & 0 & 0 & 0 & 0 & 0 & 0 & 0 \\
$\mathrm{i} 5$ & 0 & 0 & 0 & 10 & 0 & 0 & 0 & 10 & 0 & 8 & 8 & 0 & 0 & 11 & 11 \\
$\mathrm{i} 6$ & 0 & 0 & 0 & 12 & 0 & 0 & 0 & 10 & 0 & 8 & 17 & 0 & 0 & 17 & 14 \\
$\mathrm{i} 7$ & 0 & 0 & 0 & 0 & 0 & 0 & 10 & 0 & 0 & 10 & 0 & 0 & 0 & 0 & 0 \\
$\mathrm{i} 8$ & 0 & 0 & 36 & 0 & 10 & 0 & 0 & 0 & 0 & 0 & 0 & 0 & 0 & 0 & 0 \\
$\mathrm{i} 9$ & 10 & 0 & 0 & 12 & 0 & 5 & 0 & 0 & 0 & 8 & 16 & 0 & 0 & 12 & 13 \\
$\mathrm{i} 10$ & 9 & 1 & 0 & 12 & 0 & 5 & 0 & 10 & 2 & 8 & 14 & 1 & 10 & 12 & 12 \\
\hline
\end{tabular}

Table 4 Manufacturing costs of facilities for industrial case study (RMU/batch)

\begin{tabular}{rrrrrrrrrrrrrrrr}
\hline & $\mathrm{p} 1$ & $\mathrm{p} 2$ & $\mathrm{p} 3$ & $\mathrm{p} 4$ & $\mathrm{p} 5$ & $\mathrm{p} 6$ & $\mathrm{p} 7$ & $\mathrm{p} 8$ & $\mathrm{p} 9$ & $\mathrm{p} 10$ & $\mathrm{p} 11$ & $\mathrm{p} 12$ & $\mathrm{p} 13$ & $\mathrm{p} 14$ & $\mathrm{p} 15$ \\
\hline $\mathrm{i} 1$ & 1 & 1 & 0 & 10 & 0 & 3 & 0 & 1 & 1 & 1 & 3 & 1 & 0 & 1 & 1 \\
$\mathrm{i} 2$ & 10 & 0 & 0 & 5 & 0 & 2 & 0 & 5 & 0 & 10 & 2 & 0 & 2 & 5 & 2 \\
$\mathrm{i} 3$ & 0 & 0 & 0 & 0 & 0 & 0 & 0 & 0 & 0 & 0 & 0 & 0 & 1 & 0 & 0 \\
$\mathrm{i} 4$ & 0 & 0 & 0 & 1 & 0 & 0 & 0 & 0 & 0 & 0 & 0 & 0 & 0 & 0 & 0 \\
$\mathrm{i} 5$ & 0 & 0 & 0 & 20 & 0 & 0 & 0 & 20 & 0 & 20 & 20 & 0 & 0 & 5 & 20 \\
$\mathrm{i} 6$ & 0 & 0 & 0 & 10 & 0 & 0 & 0 & 10 & 0 & 10 & 10 & 0 & 0 & 1 & 10 \\
$\mathrm{i} 7$ & 0 & 0 & 0 & 0 & 0 & 0 & 10 & 0 & 0 & 10 & 0 & 0 & 0 & 0 & 0 \\
$\mathrm{i} 8$ & 0 & 0 & 1 & 0 & 5 & 0 & 0 & 0 & 0 & 0 & 0 & 0 & 0 & 0 & 0 \\
$\mathrm{i} 9$ & 10 & 0 & 0 & 10 & 0 & 10 & 0 & 0 & 0 & 10 & 8 & 0 & 0 & 1 & 10 \\
$\mathrm{i} 10$ & 15 & 15 & 0 & 15 & 0 & 15 & 0 & 15 & 15 & 15 & 15 & 15 & 15 & 15 & 15 \\
\hline
\end{tabular}

Table 5 Case study parameters

\begin{tabular}{lll}
\hline Parameter & Value & Unit \\
\hline Setup time & 14 & Days \\
Setup cost & 2 & RMU/changeover \\
Setup 'expiration' time & 90 & Days \\
Sales price & 2.5 & RMU/kg \\
Storage cost & 0.01 & RMU $/ \mathrm{kg} \times$ period $)$ \\
Storage period & 90 & Days \\
Shelf life & 2 & Years \\
Production time per year & 360 & Days \\
Backlog decay & 0.5 & Per 3 months \\
Backlog penalty & 0.1 & RMU/kg \\
\hline
\end{tabular}


structed from the permutation by a construction heuristic, often Giffler-Thompson, which iteratively selects the job with the highest priority (lowest permutation index) from the set of eligible jobs, and then schedules it at the earliest possible time. This avoids infeasible solutions and introduces a desirable heuristic bias, in the sense that it excludes obviously bad solutions (such as schedules with big gaps) from the search space. Inspired by this work, we also propose using an indirect, permutation-based encoding combined with a construction heuristic. The construction heuristic, however, had to be carefully designed for the problem at hand.

In the following two subsections, we first explain the proposed construction heuristic, then provide details on the GA used.

\subsection{Construction heuristic}

The construction heuristic works on the basis of forecasted demands, in our case demands for each year and product (see Table 1). Its task is to schedule demands (or rather the production to satisfy the demand, but for simplicity we will continue calling it demand) sequentially, in the order prescribed by the GA. When deciding at what time and what facility to insert a new demand into the schedule, the heuristic explores a number of different alternatives, and then greedily picks the alternative that creates the smallest additional cost. In essence, the heuristic will consider each facility in which the product may be produced. It then tries to schedule the entire demand in an uninterrupted way as late as possible to minimise storage cost, and as late as possible but adjacent to already scheduled demand of the same product to avoid setup cost and time.

Only if these alternatives are not feasible for a facility, e.g., because a facility does not have a sufficiently large gap in its schedule, further options are explored that either move some of the already scheduled demands to make sufficient space for the new demand, split the demand into two parts and schedule the second part in another facility, or backlog the demand.

Figure 1 provides a simple example based on just two facilities and four products, while Algorithm 1 lays out brief pseudocode of the construction heuristic. The six alternatives considered shall now be explained in detail.

(I) Schedule as late as possible The first alternative considered is to schedule the entire demand as late as possible but before the due date, as one uninterrupted block, which minimizes storage cost at this facility. In the example, this is possible for Facility 1, see Fig. 1 (I), but not for Facility 2, since there is not sufficient uninterrupted capacity available to schedule the entire demand.

(II) Schedule next to previous demand To avoid setup times and setup costs, it may be beneficial to schedule a demand adjacent to the same product already scheduled. The heuristic picks the latest time slot before the due date that allows to link to a previously-scheduled demand of the same product, and has sufficient available capacity to schedule the entire demand-see Fig. 1 item (II). Again, this is only possible on Facility 1, as Facility 2 does not have sufficient uninterrupted capacity. Note that due to the avoided setup time, the overall time required to produce the demand is smaller. 


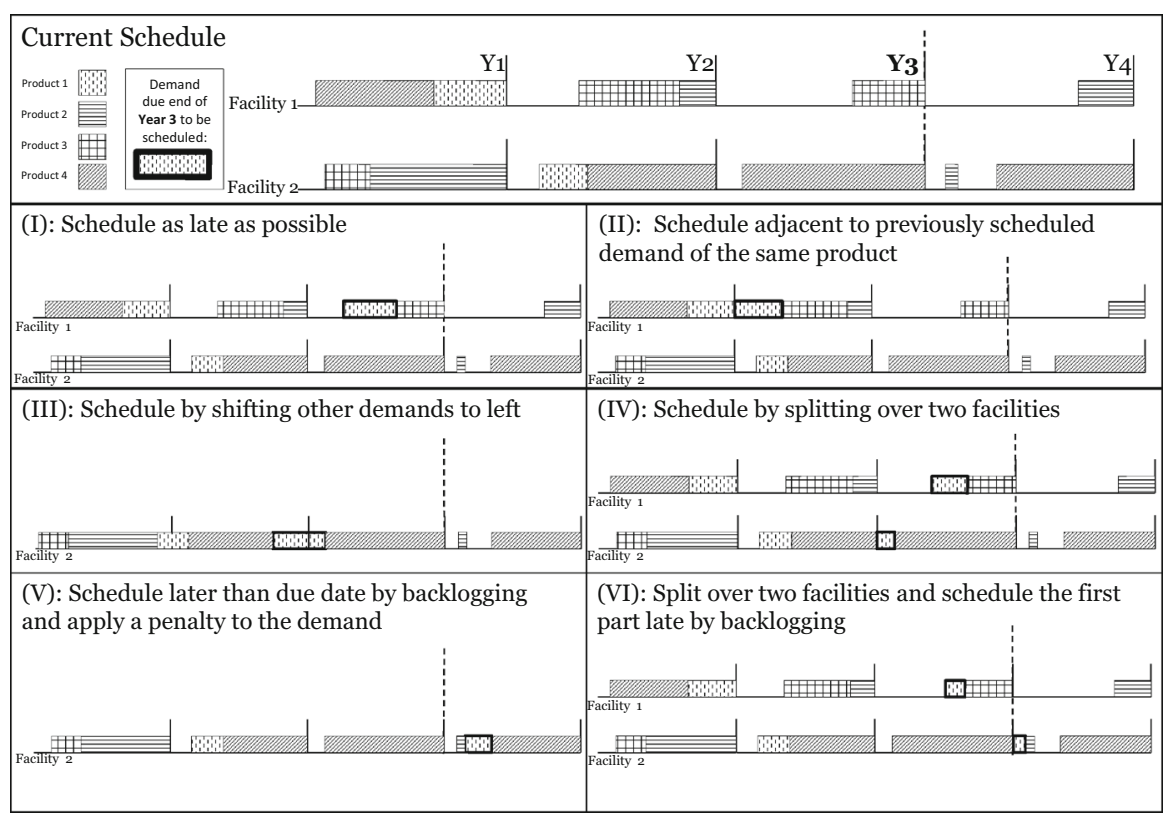

Fig. 1 Visualisation of construction heuristic, based on a simple example with two facilities and four demands. Items $(I)-(V I)$ show the alternatives the heuristic considers when identifying the most profitable place to insert a new demand into the schedule. The rectangle representing a demand includes the setup time (so length varies depending on where the new demand is inserted). Note that just using (I) or (II), feasible options are found on Facility 1 but not on Facility 2. Therefore the heuristic will terminate its search on Facility 1 but continue on Facility 2 using (III), (IV), (V), and (VI)

If, in a particular facility, none of the above two alternative insertion attempts resulted in a feasible solution, the following options are explored. In particular, it is attempted to move already scheduled demands, to split a demand, and to backlog a demand.

(III) Move previously scheduled demands Since there was not a sufficiently long gap in the current schedule to allocate the entire production for the new demand, one possibility to create a feasible schedule may be to shift previously-scheduled demands to an earlier time to make space for the new demand. Thereby, the heuristic identifies the latest gap in the considered facility before the due date. All conflicting scheduled demands before this gap are shifted backward in time (towards the start of the planning horizon), without changing the order, and just enough to make space for the new demand. This can be seen in Fig. 1 item (III) for Facility 2, where 4 previously scheduled demands had to be left-shifted to make space for the new demand.

(IV) Split demand Another option to fit the demand may be to split the new demand. In this alternative, the heuristic will again consider the latest gap before the due date, and use all available consecutive capacity. Then, it will attempt to schedule the rest of the demand at each of the other facilities, but only considering options $(I),(I I)$, and $(V)$ (which is described below). An example is provided in Fig. 1 


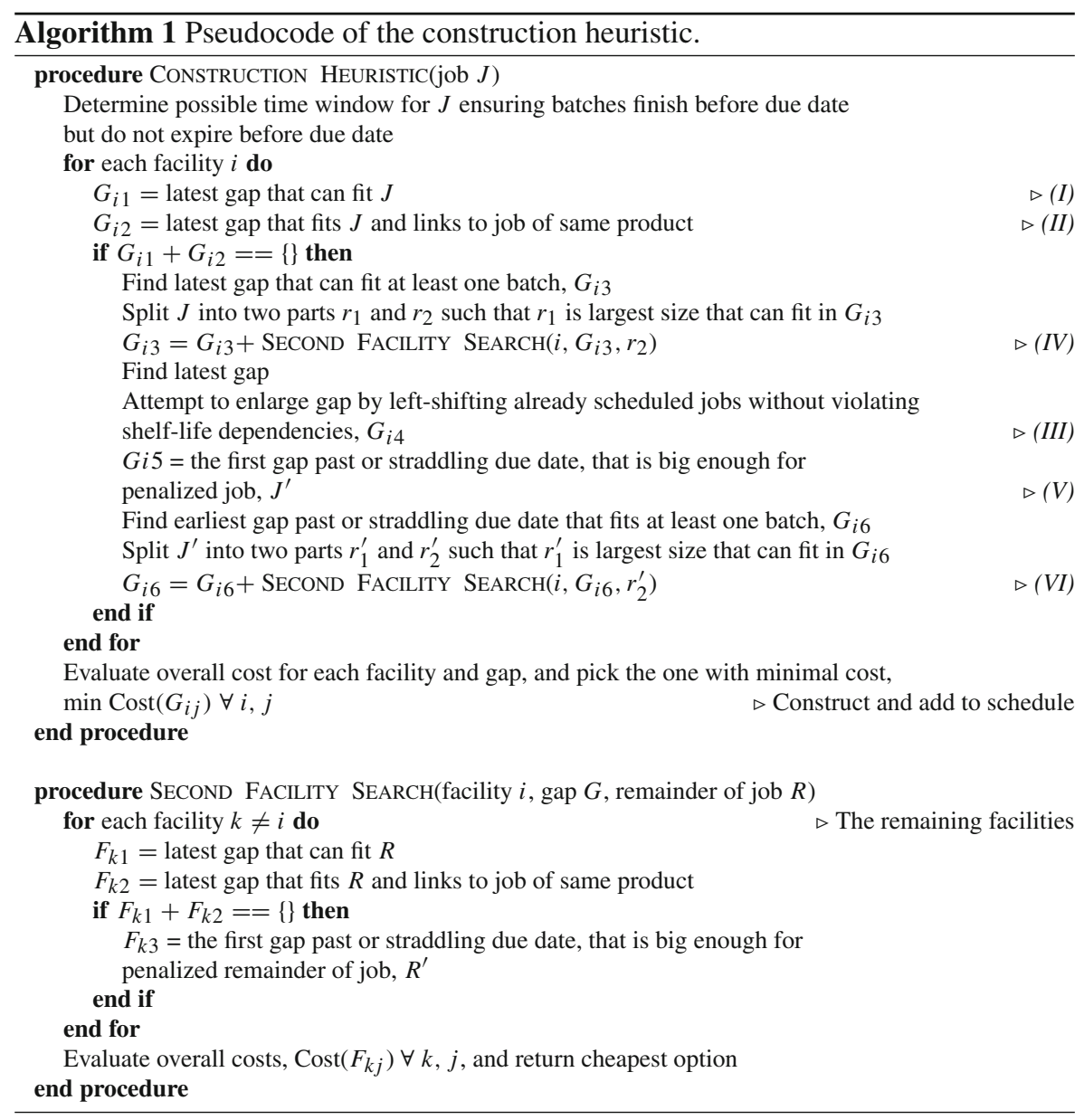

item $(I V)$, where only a small fraction of the demand can be scheduled at Facility 2 , and the remainder is then moved to Facility 1 . Note that splitting the demand may cause an additional setup time and setup cost. A demand can only be split into two i.e., a demand cannot be split more than once.

(V) Backlog If the facilities are really busy, it may be best (or the only feasible option) to backlog the demand. That is to say that the time slot allocated to produce the material to meet the demand falls partly or wholly later than the due date for the demand. As described in the case study, this will result in a monetary penalty and part of the demand being lost, as is reflected in Fig. $1(\mathrm{~V})$ by the smaller rectangle for the scheduled demand). In order to reduce the magnitude of the penalty, the heuristic will schedule the demand as early as possible in a gap that either straddles, or is later than, the due date. An example is provided in Fig. 1 item $(V)$.

(VI) Backlog and split As a kind of last resort, with this alternative, the heuristic will combine steps $(I V)$ and $(V)$. As in $(I V)$, the demand is split, but rather than using 
the latest gap before the due date, the first part of the demand is scheduled in the earliest gap after the due date. The remaining portion of the demand is attempted again to be scheduled in all other facilities, but only using options $(I),(I I)$ or $(V)$. This is illustrated in Fig. 1 item (VI).

The above alternatives will be evaluated for all the facilities that are capable of producing the product. Then, the demand is inserted into the schedule according to the most profitable alternative examined, and the algorithm moves on to schedule the next demand.

Overall, if there are $n$ facilities, in the worst case the heuristic considers $6 n^{2}-2 n$ alternatives: $(n)$ alternatives for each of the options $(I),(I I),(I I I)$ and $(V)$, and then $3 n(n-1)$ alternatives each for option $(I V)$ and option (VI), due to different possibilities in scheduling the remaining part of a demand in case of a split. This means that in the worst case the complexity of the construction heuristic is $O\left(m n^{2}\right)$, where $m$ is the number of demands and $n$ the number of facilities. In practice, however, as we will show later, in the majority of cases, only options (I) and (II) are explored per facility.

Note that batch production means that unless the demand is exactly equal to an integer multiple of the batch size (which itself is different for different facilities) it is not possible to produce exactly the required demand. In such cases, the number of produced batches is always rounded up to the minimal integer number of batches necessary to fulfill the demand. The amount overproduced in such a case is put in storage, possibly to be used to (partly) fulfill future demand. Before going through the steps above to insert a demand into the schedule, the construction heuristic will always check whether the product is in the storage, and try to partially fulfill the demand from storage. The cost associated with this is storage cost only, as manufacturing costs are invoked at the time of production, i.e., when a previous scheduled demand produced that overcapacity. Products left in storage that the heuristic can not use in later steps are considered lost and have no value.

\subsection{Genetic algorithm}

The quality of the solution produced by the above construction heuristic is to some extent dependent on the order in which the demands are inserted into the schedule since available production capacity is more restricted the later a demand is considered. By giving priority to certain demands mainly three situations can be created.

1. Demands of the same product that should be ideally scheduled consecutively to avoid setup costs, can be assigned similar priorities, making it very likely that the construction heuristic will link them together.

2. Demands that are best scheduled just before the due date to save storage cost can be given a high priority. This will lead to the construction heuristic scheduling these demands early on, at a time where still a lot of capacity is available, and the cheapest option just before the due date would be selected.

3. Demands that benefit most from a highly utilised facility (e.g., because all other facilities are much more expensive), can also be given high priority, which will lead to early scheduling when this highly demanded facility is still available. 
Table 6 Profit performance for base case (in RMU) \pm SD for three different population sizes and mutation rates

\begin{tabular}{llll}
\hline Population size & \multicolumn{3}{l}{ Mutation rate } \\
\cline { 2 - 4 } & 0.01 & 0.02 & 0.03 \\
\hline 20 & $66612 \pm 0.9$ & $66601 \pm 1.0$ & $66594 \pm 0.9$ \\
30 & $66613 \pm 0.8$ & $66604 \pm 0.9$ & $66593 \pm 0.9$ \\
60 & $66612 \pm 0.8$ & $66603 \pm 0.8$ & $66592 \pm 0.8$ \\
\hline
\end{tabular}

Optimising this order is left to the GA, which was implemented in Java using the ECJ library (Luke et al. 2014). It uses a permutation representation of all the demands to be scheduled, i.e., 225 in the industrial case study used here (the number of elements in Table 1). Specifically, each demand is given a unique ID number (from 1 to 225), and the chromosome is a permutation of these numbers. The ordering of the numbers on the chromosome determines the order by which the construction heuristic processes the respective demands (from first to last position) and thus influences the resulting schedule.

Originally, we initialised individuals randomly, but then realised that better solutions are produced if demands from a single year are grouped together on the chromosome. Unless stated otherwise, the results in this paper are thus based on runs where $50 \%$ of the population is initialised randomly, whereas the other $50 \%$ only randomise the sequence of demands from the same year, but maintain the sequence of years (i.e., all demands of a particular year appear in the permutation before the demands of later years). For fitness evaluation, the GA calls the construction heuristic described in Sect. 4.1 which builds a schedule by inserting demands iteratively in the order prescribed by the solution's chromosome. The actual fitness is then the overall profit of the resulting schedule, i.e., revenue minus storage, production, setup cost and backlog penalty. This objective function is defined mathematically in the "Appendix". We did not spend much effort on tuning parameters to this problem, but experiments with different population sizes and mutation rates show that results are rather insensitive to the parameter settings (see Table 6). For the rest of the paper, we used a population size of 30 , generational reproduction with elite of 6 , and fitness proportional selection with stochastic universal sampling. For crossover, we used the Precedence Preserving Crossover (PPX) proposed by Bierwirth et al. (1996) which ensures that if a demand $i$ is before a demand $j$ in both individuals, this will also be true in the offspring. As the mutation operator we used shift mutation, which iterates through every element of the permutation and, with probability $p_{m}=0.02$, removes a demand and re-inserts it at a new random position. The algorithm is run for 1500 generations, and all results are based on averages over 50 runs.

\section{Empirical evaluation}

\subsection{Comparison with mathematical programming}

We ran our algorithm on the case study described in Sect. 3, and results for this are reported in Table 7 as "standard case/GA". As it turns out, the case study has ample 
Table 7 Profit, customer service level (CSL), and other characteristics for GA and MILP

\begin{tabular}{|c|c|c|c|c|c|c|}
\hline & \multicolumn{2}{|c|}{ "Standard case" } & \multicolumn{2}{|l|}{$2 \times$ Demand } & \multicolumn{2}{|l|}{$3 \times$ Demand } \\
\hline & GA & MILP & GA & MILP & GA & MILP \\
\hline Revenue & $\mathbf{7 4 , 5 3 3}$ & $74,490.9$ & $\begin{array}{r}\mathbf{1 4 8 , 9 5 2} \\
\pm 18.3\end{array}$ & $148,389.8$ & $\begin{array}{r}\mathbf{2 2 2 , 5 3 0} \\
\pm 89.8\end{array}$ & 221,603 \\
\hline $\begin{array}{l}\text { Manufacturing } \\
\text { costs }\end{array}$ & $\begin{array}{l}\mathbf{7 2 7 4} \\
\quad \pm 0.69\end{array}$ & 7452 & $\begin{array}{l}\mathbf{2 0 , 3 6 7} \\
\quad \pm 25.12\end{array}$ & 20,541 & $\begin{array}{l}42,427 \\
\quad \pm 83.7\end{array}$ & 40,346 \\
\hline Storage costs & $\mathbf{3 3 7} \pm 0.9$ & 447.4 & $\mathbf{8 6 2} \pm 4.8$ & 952.8 & $\begin{array}{l}1698 \\
\pm 11.7\end{array}$ & 1559.9 \\
\hline Setup costs & $318 \pm 0.9$ & 272 & $330 \pm 1.20$ & 274 & $342 \pm 1.56$ & 276 \\
\hline $\begin{array}{l}\text { Backlog } \\
\text { penalties }\end{array}$ & $\mathbf{0} \pm 0.0$ & 3.3 & $\mathbf{9} \pm 1.5$ & 53.4 & $\mathbf{8 8} \pm 7.5$ & 156.7 \\
\hline Profit & $\begin{array}{r}\mathbf{6 6 , 6 0 4} \\
\pm 0.9\end{array}$ & 66,316 & $\begin{array}{r}\mathbf{1 2 7}, \mathbf{3 8 5} \\
\pm 11.0\end{array}$ & $126,568.7$ & $\begin{array}{r}177,975 \\
\pm 52.6\end{array}$ & 179,265 \\
\hline CSL & $100 \%$ & $99.9 \%$ & $\begin{array}{c}\mathbf{9 9 . 9 \%} \pm \\
0.01 \%\end{array}$ & $99.5 \%$ & $\begin{array}{l}\mathbf{9 9 . 5 \%} \\
\quad \pm 0.04 \%\end{array}$ & $99.1 \%$ \\
\hline Time (s) & 105.1 & 600.5 & 184.3 & 600.4 & 269.5 & 600.4 \\
\hline Optimality gap & - & $0.25 \%$ & - & $0.64 \%$ & - & $0.92 \%$ \\
\hline
\end{tabular}

For the GA, mean \pm SD are listed, whereas MILP is a deterministic method and was only run once. Best mean is highlighted in bold-where the difference is not significant both are highlighted. GA timing values are the average time elapsed for each of the 50 runs (i.e., the total runtime of 50 runs divided by 50)

production capacity, which is due to modelling the option of outsourcing production at higher cost as additional facilities. To see how our algorithm would perform also in a more loaded scenario, we also tested variations of the case study where we increased the demand in each year by a factor of 2 or 3, and the results of these experiments are reported in Table 7 as well.

To judge the performance of our proposed algorithm, we compare it with a mixed integer linear programming (MILP) implementation as described by Lakhdar et al. (2007) and replicated in the "Appendix". We re-implemented the approach and compared the results of the GA with the results we obtained with our mathematical programming implementation. This ensures that the solutions are generated with exactly the same assumptions and data. However, there is one important difference that deserves discussion. The MILP model has variables that specify how much is produced for each facility, product and time period. It thus requires the problem to be broken down into discrete time periods, and it allows for at most one product to be produced in a particular facility and time period. The choice of the length of a time period is somewhat arbitrary, but has huge implications. If the time period is chosen very large, then most demands would require only a fraction of a time period to be produced, the facility would be idle in the remaining part of the time period, leading to poor solutions. On the other hand, if the length of a time period is chosen to be rather short, because the number of batches to be produced is integer, often a fraction of the time period remains unused (e.g., if a time period is 5 days, and producing a batch takes 3 days, only one batch can be produced in each time period and 2 days in each time period remain unused-up to the point where a time period is too short for 
even one batch and there is no feasible solution). Furthermore, reducing the length of the time period increases the number of variables and constraints quite significantly, with corresponding drastic implications on running time. After some experimenting, we concluded that the 90 day period used by Lakhdar et al. (2007) indeed performs well, and all our results are based on this time granularity.

Different from the MILP model, our GA can work with arbitrary time periods, and even continuous time, without any implications on running time. In our implementation, we chose to use days as the smallest time unit. This allows us to model reality more closely than the mathematical programming implementation. As a result, the GA sometimes is able to produce solutions with a higher profit than the MILP approach, even if MILP is run to optimality. Please note that we do not claim that the MILP implementation by Lakhdar et al. (2007) is the best possible, or that it is not possible to design an MILP formulation that circumvents or at least reduces the impact of the time period length. However, the MILP model is the only one we found in the literature for this problem, and we do believe that one of the advantages of GAs is their greater flexibility in modelling the real world, and that solving a problem heuristically that is close to reality can sometimes work better than a model further from reality but solved to optimality.

Results from the MILP model and the GA are compared in Table 7. As can be seen, in the standard case as taken from Lakhdar et al. (2007), the GA solution has lower manufacturing costs, i.e., utilises better the low-cost facilities, and lower storage costs. It also manages to satisfy all the demand (customer service level of 100\%), whereas the MILP model chooses to backlog some of the demand. This is because the GA tries to satisfy all the demand as first priority and only backlogs if there is no other feasible option. The MILP, however, has an explicit trade-off between backlog and other costs, and backlogs if the resulting solution has a higher profit. On the other hand, the setup costs of the GA solution are higher. Overall, the profit generated by the GA solution is consistently higher, and by more than the $0.25 \%$ optimality gap, i.e. the difference between the best solution found and the upper bound determined by the MILP solver. This is possible because MILP, due to its imposed time granularity, has an artificially restricted search space. It can switch less often between products, resulting in lower setup cost and higher storage cost. Also, it sometimes wastes part of a time period, which may mean the need to use occasionally more expensive facilities, resulting in higher manufacturing costs. These differences can be seen also by comparing the Gantt charts of the optimal solutions found by the MILP and the GA which are depicted in Fig. 2. The Gantt chart of the MILP solution generally shows shorter campaigns (sequences of batches of the same product), and, especially visible on facility i4, small gaps between production in different time periods, simply because the time period (of 90 days) is not equivalent to a duration spanned by a multiple of batches for this product in this facility. The schedule optimised by the GA has longer un-interrupted idle time, which may be advantageous if a new product is introduced to the facility or if a third party is seeking to rent and use production capacity.

For the scenario with twice the demand, the conclusions are similar to the base case. However, for three times the demand, it seems backlogging becomes crucial, and the MILP approach seems better in doing that. While backlogging reduces the products sold due to lost demand and thus reduces revenue, the savings that can be 

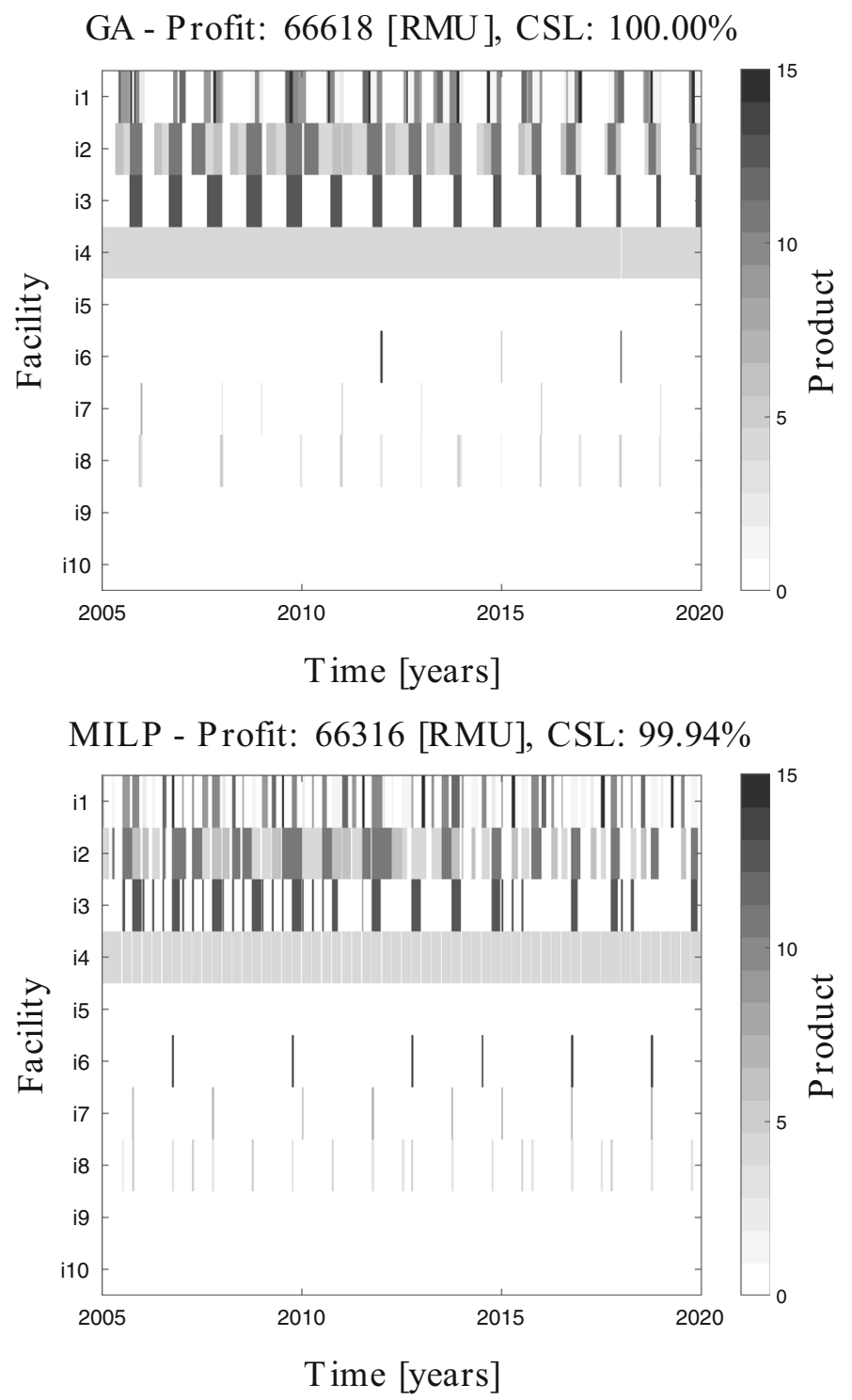

Fig. 2 Exemplary Gantt chart of a schedule generated by the GA (top) and MILP (bottom), for the standard case. The profit and customer service level (CSL) for each schedule is also indicated

achieved in terms of manufacturing cost and setup cost seem to outweigh this loss, and the overall profit of the MILP approach is higher in this scenario. Whether a slightly higher profit justifies a lower customer service level is a different issue. The GA's construction heuristic, always tries to meet all the demand, even if this may lead to a possibly lower profit. Finally, we observe that the GA has still lower storage cost and higher setup cost, probably due to not being constrained by the coarse time periods. 
Table 8 Runtime of MILP until it reached an optimality gap of $0.25 \%$, and runtime of GA to reach the same solution quality as was reached by MILP, for different problem sizes, depending on problem size

\begin{tabular}{llll}
\hline & 15 years & 23 years & 30 years \\
\hline Target (RMU) & 66,284 & 90,236 & 111,229 \\
MILP time (s) & 200.86 & 824.134 & 1332.59 \\
GA time (s) & 0.07 & 0.131 & 0.195 \\
\hline
\end{tabular}

Runtimes strongly depend on the implementation skills of the developer, the hardware used, and software tools used, and thus have to be handled with caution. Nonetheless, Table 7 also reports on the runtime of the two algorithms. For MILP, the stopping criterion was 600s, so the runtime remained the same, but the optimality gap increased as the problem became more difficult by increasing the demand and thus utilisation level. The GA was run for a fixed number of generations. The computational time still increased with increasing the demand level. The reason is that an increasing demand raises the utilisation level and the construction heuristic is then less likely to be able to schedule a demand in steps (I) or (II), and thus more often has to look at the other alternatives for scheduling it. This will be explored further in the next subsection.

We also investigated the scaling behaviour of both optimisation methods with increasing problem sizes. For this, we ran the GA and MILP for problems with longer time horizons of 23 and 30 years (in addition to the 15 year-long base case). For the longer time horizons, the demand forecasts for the years after year 15 were set equal to the forecast for each product in year 15. To compare the two methods, the MILP was first run for all three problem sizes with a stopping criterion of a $0.25 \%$ optimality gap, at which point, the solution quality (profit) was recorded along with the time taken to achieve the solution. This profit value was then used as a target for the GA. The average time over the 50 runs that it took for the GA to match or beat those targets was recorded. The results of this experiment are shown in Table 8. As can be seen, the time required for the MILP and GA increases roughly linearly with problem size, however the factor by which runtime increases when moving from 15 to 30 years is 6.6 for MILP, but only 2.8 for the GA.

Overall, from these results, we conclude that the suggested GA approach is competitive with the MILP approach, but does not suffer from the introduction of artificial time periods and thus is sometimes able to find better solutions than MILP. The trend seems to be that the times for both optimisation methods are going up linearly. However the increase of the GA approach is of a smaller factor than that of the MILP optimisations. This suggests that the relative performance of the GA is less susceptible to the detrimental impact of increasing the scale of the problem.

\subsection{Algorithm components}

In order to better understand the importance and robustness of the various components of our algorithm, we did some additional experiments. 
Table 9 Breakdown of how often each part of the heuristic is used in optimised solutions, mean \pm SD

\begin{tabular}{llll}
\hline & $1 \times$ Demand & $2 \times$ Demand & $3 \times$ Demand \\
\hline$(I)$ & $71.9 \% \pm 0.18 \%$ & $70.7 \% \pm 0.27 \%$ & $64.1 \% \pm 0.25 \%$ \\
$(I I)$ & $20.9 \% \pm 0.19 \%$ & $16.8 \% \pm 0.22 \%$ & $12.8 \% \pm 0.20 \%$ \\
$(I I I)$ & $0.3 \% \pm 0.03 \%$ & $2.8 \% \pm 0.13 \%$ & $4.9 \% \pm 0.14 \%$ \\
$(I V)$ & $6.9 \% \pm 0.03 \%$ & $8.3 \% \pm 0.17 \%$ & $10.4 \% \pm 0.12 \%$ \\
$(V)$ & $0.0 \%$ & $1.3 \% \pm 0.18 \%$ & $7.7 \% \pm 0.22 \%$ \\
$(V I)$ & $0.0 \%$ & $0.0 \%$ & $0.04 \% \pm 0.02 \%$ \\
Total backlogged jobs & $0.0 \%$ & $1.8 \% \pm 0.25 \%$ & $9.2 \% \pm 0.25 \%$ \\
\hline
\end{tabular}

Also detailed is the percentage of separate jobs that are delivered late

Fig. 3 The ratio in profit of the full model compared to the simple model, optimized by the GA, shown for multiples of the base market demand as presented in the case study. The increasing ratio indicates the increasing benefit the full model will have in more complicated scheduling problems than the basic case study. Error bars represent the standard error

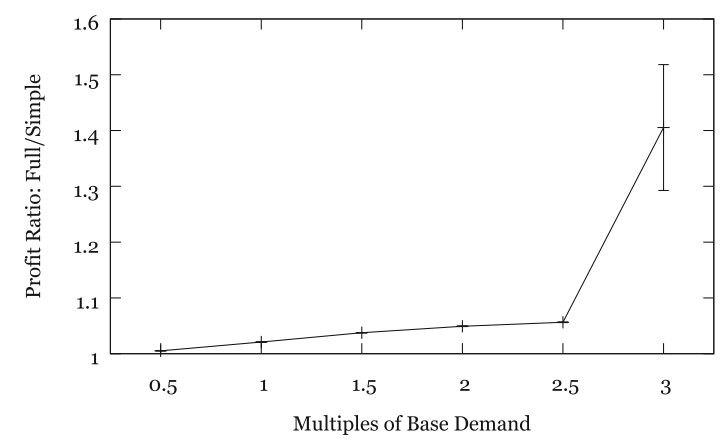

Table 9 examines how often the various alternatives to insert a demand are actually selected by the construction heuristic, averaged over the best solution found in each of the 50 runs. As can be seen in the table, in the standard case, the majority of demands $(92.8 \%)$ are inserted by either scheduling it as late as possible (I), or adjacent to a previous demand of the same type (II). This is reassuring, since if such an insertion is possible, the other options are not tested, which significantly speeds up the algorithm. As we move to the scenarios with higher demand, the percentage drops from 92.8 to $78.9 \%$. This still constitutes the majority of cases, but clearly the other insertion alternatives of the heuristic become more important.

Figure 3 looks at the relevance of the alternatives (III)-(VI) in terms of their impact on profit. It shows the ratio of the obtained profit depending on whether the construction heuristic during the GA search was limited to looking at alternatives $(I)+(I I)$ (denoted as "Simple"), or all alternatives ("Full"). A profit ratio of 1 means that the two models obtain the same profit, while a greater profit ratio indicates that the full model is able to achieve higher profits than the simple model. It confirms that the more complicated cases with splitting, backlogging and moving previously scheduled demand are responsible for an increasing share of the profit as the overall demand is increased. Especially once the demand is increased to three times the original values, there seems to be a step change and the more complicated alternatives seem to become indispensable. 


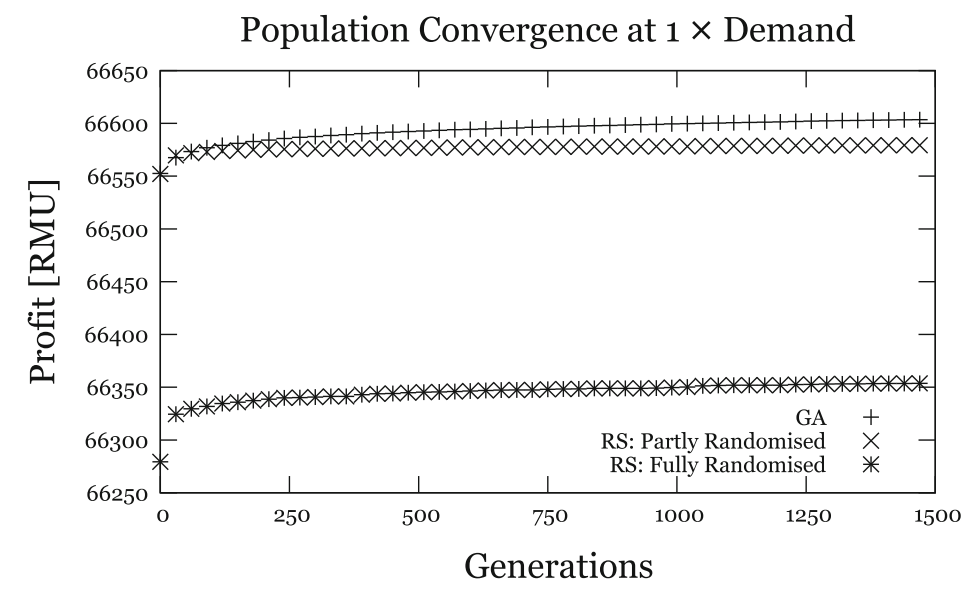

Population Convergence at $2 \times$ Demand

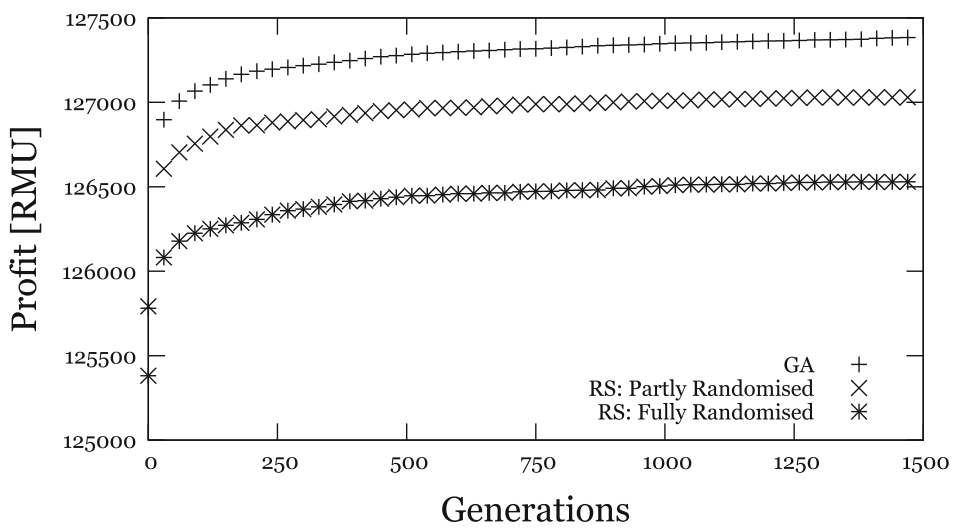

Population Convergence at $3 \times$ Demand

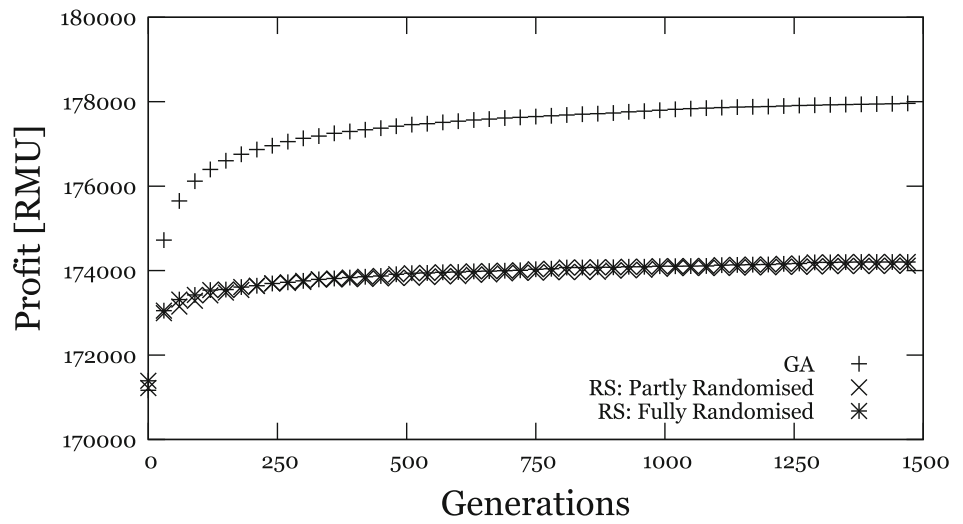

Fig. 4 Convergence of profit over generations, for GA and random search. Random search is tested with fully randomised permutations and where $50 \%$ of the permutations are randomised only amongst demands of the same year, but keep the order on years. $R S$ random search 
Lastly, Fig. 4 shows the convergence of the GA over generations, and compares it with a purely random search, using fully random permutations or limited random permutations, i.e., when half of the permutations are only random amongst demands of the same year, but the order on years is kept. As can be seen the results optimised by the GA are considerably better than the results obtained by random search. The limited randomisation helps in particular for the less loaded problems $(1 \times$ Demand $)$, but is no longer better than fully randomised permutations for the case of $3 \times$ Demand. This also makes sense, as with higher utilisation of the facilities, there is increasing need to schedule demands outside the year the demand is delivered, and the artificial limitation of randomisation to within a year is no longer helpful.

\section{Conclusion}

In this paper, we have considered the lot sizing and scheduling problem for a complex biopharmaceutical production scenario featuring multiple products, multiple facilities, and batch processing. For this challenging optimisation problem, we have proposed an GA based on an indirect permutation encoding that is decoded into a full schedule by a novel construction heuristic tailored to the problem at hand. A comparison with an MILP approach from the literature showed that the GA is at least competitive, and often produces even better results than the MILP approach. The reason is that the MILP model artificially imposes a time granularity by dividing the time into discrete periods that is not needed in the GA approach. This shows that although GAs are heuristic methods, they can sometimes outperform exact methods not only in terms of running time, but also because they are able to work with a model closer to reality.

In the future, we are considering various extensions of the proposed GA. First, in reality, demand is estimated and uncertain, so we would like to adapt our approach to stochastic and dynamic problems. This would also hopefully be a good juncture to investigate different instances of the problem solved in this work. Second, often in biopharmaceutical production, other objectives such as risk play a role, and an extension of our approach to the multi-objective case seems straightforward. Third, we plan to capture more realistic biopharmaceutical processes, e.g., by modelling multiple production stages. Fourth, the biopharmaceutical industry has seen a resurgence of interest in alternative ways to batch manufacturing, in particular continuous manufacturing, and so we would like to extend our algorithmic framework to also be applicable to this form of manufacturing. Fifth, from a theoretical perspective, it would be important to investigate the formal properties of the proposed GA to, for example, guarantee that the optimal schedule is indeed within reach of the search algorithm. Finally, one might explore also other optimisation methodologies to solve this problem such as constraint programming (Laborie 2009) or hybrid approaches (Blum and Raidl 2016).

Acknowledgements The first author would like to acknowledge funding through the EPSRC Centre for Doctoral Training in Emergent Macromolecular Therapies, EP/L015218/1.

Open Access This article is distributed under the terms of the Creative Commons Attribution 4.0 International License (http://creativecommons.org/licenses/by/4.0/), which permits unrestricted use, distribution, 
and reproduction in any medium, provided you give appropriate credit to the original author(s) and the source, provide a link to the Creative Commons license, and indicate if changes were made.

\section{Appendix}

The appendix summarises the mathematical programme used in this paper and introduced by Lakhdar et al. (2007).

\section{Notation}

The indices $i, p$, and $t$ denote individual facilities, products, and time periods respectively. The subsets characterising the facilities being considered are: $P I_{i}$, the set of products produced by facility $i ; I P_{p}$, the set of facilities that can produce product $p$; and $T I_{i}$, the set of time periods in which facility $i$ is available for use.

\section{Binary variables}

$Y_{i p t} \quad 1$ if product $p$ is produced over period $t$ at facility $i ; 0$ otherwise

$Z_{i p t} \quad 1$ if a new campaign of product $p$ at facility $i$ is started in period $t ; 0$ otherwise

\section{Integer variables}

$B_{i p t} \quad$ amount of product $p$ produced over period $t$ at facility $i$, batches

\section{Continuous variables}

$I_{p t} \quad$ amount of product $p$ stored over period $t$, kilograms

$K_{i p t} \quad$ amount of product $p$ produced over period $t$ at facility $i$, kilograms

Prof expected operating profit, RMU

$S_{p t} \quad$ amount of product $p$ which is sold over period $t$, kilograms

$T_{i p t} \quad$ production time for product $p$ at time period $t$ at facility $i$

$T f_{i t}^{t o t} \quad$ total production time over period $t$ at facility $i$

$W_{p t} \quad$ amount of product $p$ wasted over period $t$, kilograms

$\Delta_{p t} \quad$ amount of product $p$ which is late over period $t$, kilograms

\section{Parameters}

$C_{p} \quad$ storage capacity of product $p$, kilograms

$D_{p t} \quad$ demand of product $p$ at time period $t$, kilograms

$r_{i p} \quad$ production rate of product $p$ at facility $i$, batches per unit time

$H_{t} \quad$ available production time horizon over time period $t$

$T_{i p}^{\max } \quad$ maximum production time for product $p$

$T_{i p}^{\min } \quad$ minimum production time for product $p$

$y d_{i p} \quad$ yield conversion factor, kilograms per batch

$\alpha_{i p} \quad$ lead time for production of first batch of product $p$ at facility $i$

$\zeta_{p} \quad$ life time of product $p$, number of time periods $t$

$v_{p} \quad$ unit sales price for each kilogram of product $p$, RMU per kilogram

$\eta_{i p} \quad$ unit cost for each batch produced of product $p$ in facility $i$, RMU per batch

$\psi_{p} \quad$ unit cost for each new campaign of product $p, \mathrm{RMU}$ 
$\delta_{p} \quad$ unit cost charged as penalty for each late kilogram of product $p$, RMU per kilogram

$\rho_{p} \quad$ unit cost for each stored kilogram of product $p$, RMU per kilogram

$\pi \quad$ rate of backlog decay

\section{Production constraints}

Constraint (1) represents batch processing. The number of batches produced in facility $i$ of product $p$ at time period $t, B_{i p t}$, is determined by a continuous production rate, $r_{i p}$, production lead time, $\alpha_{i p}$, and production time $T_{i p t}$. The lead time allows for the duration of the first batch of a campaign plus the setup and cleaning time before the first batch commences. Incorporation of lead time is enforced by a binary variable $Z_{\text {ipt }}$.

$$
B_{i p t}=Z_{i p t}+r_{i p}\left(T_{i p t}-\alpha_{i p} Z_{i p t}\right) \quad \forall i, \quad p \in P I_{i}, \quad t \in T I_{i}
$$

Constraint (2) converts the number of batches into kilograms produced using a yield conversion factor $y d_{i p}$ which differs for each combination of facility and product. Lead time is only avoided in a facility if the same product is manufactured in the preceding period; this is covered in (3). Constraint (4) ensures that at most one product $p$ is manufactured in any given facility $i$ per time period $t$.

$$
\begin{aligned}
K_{i p t} & =B_{i p t} y d_{i p} \quad \forall i, \quad p \in P I_{i}, \quad t \in T I_{i} \\
Z_{i p t} & \geq Y_{i p t}-Y_{i p, t-1} \quad \forall i, \quad p \in P I_{i}, \quad t \in T I_{i} \\
\sum_{p \in P I_{i}} Y_{i p t} & \leq 1 \quad \forall i, \quad t \in T I_{i}
\end{aligned}
$$

\section{Timing constraints}

Constraints (5) and (6) represent the appropriate minimum and maximum production time constraints. These are only active if $Y_{i p t}$ is equal to 1 , otherwise the production times are forced to 0 .

$$
\begin{aligned}
T_{i p}^{\text {min }} Y_{i p t} & \leq T_{i p t} \quad \forall i, \quad p \in P I_{i}, \quad t \in T I_{i} \\
T_{i p t} & \leq \min \left\{T_{i p}^{\max }, H_{t}\right\} Y_{i p t} \quad \forall i, \quad p \in P I_{i}, \quad t \in T I_{i}
\end{aligned}
$$

\section{Storage constraints}

The following constraints enforce an inventory balance for production and force total production to meet product demand. In (7), the amount of product $p$ stored at the end of the time period, $I_{p t}$, is equal to the amount stored in the previous period, plus the total amount produced across all facilities $i$, less the amount sold, $S_{p t}$, and the amount of product wasted, $W_{p t}$, in the current time period $t$. Product stored cannot be negative and should not exceed maximum product storage capacity in (8); and total inventory 
at any point cannot exceed the global storage capacity in (9).

$$
\begin{aligned}
I_{p t} & =I_{p, t-1}+\sum_{i} K_{i p t}-S_{p t}-W_{p t} \quad \forall p \in P I_{i}, \quad t \in T I_{i} \\
0 & \leq I_{p t} \leq C_{p} \quad \forall p, t \\
0 & \leq \sum_{p} I_{p t} \leq C_{P}^{t o t} \quad \forall t
\end{aligned}
$$

The duration a product can be stored in inventory is limited by its shelf-life in (10).

$$
I_{p t} \leq \sum_{\theta=t+1}^{t+\zeta_{p}} S_{p \theta} \quad \forall p, t
$$

\section{Backlog constraints}

A penalty is incurred for every time period $t$ that a given amount of product $p$ is late. For a given product $p$ at time $t$, the amount of product that is late, $\Delta_{p t}$, is equal to the amount of undelivered product from the previous time period, $\Delta_{p, t-1}$, multiplied by a factor, $\pi_{p}$ (which allows for the backlog to decay), plus demand at time $t, D_{p t}$, less the sales at time $t, S_{p t}$.

$$
\Delta_{p t}=\pi_{p} \Delta_{p, t-1}+D_{p t}-S_{p t} \quad \forall p, t
$$

\section{Objective function}

The objective function is to maximise profit, which is the difference between total revenue (sales in kilogram times price $v_{p}$ ), and total operating costs which include the changeover cost at $\psi_{p}$ per setup, storage cost at $\rho_{p}$ per kilogram of product, late delivery penalties of $\delta_{p}$ per kilogram of product, and batch manufacturing cost at $v_{i p}$ for every product-facility combination. All costs and prices are in relative monetary units (RMU).

$$
\max \quad \text { Profit }=\sum_{p} \sum_{t \in T I_{i}}\left(v_{p} S_{p t}-\rho_{p} I_{p t}-\delta_{p} \Delta_{p t}-\sum_{i \in I P_{p}}\left(\eta_{i p} B_{i p t}+\psi_{i p} Z_{i p t}\right)\right)
$$

\section{References}

Almada-Lobo, B., James, R.J.: Neighbourhood search meta-heuristics for capacitated lot-sizing with sequence-dependent setups. Int. J. Prod. Res. 48(3), 861-878 (2010)

Almada-Lobo, B., Klabjan, D., Antónia carravilla, M., Oliveira, J.F.: Single machine multi-product capacitated lot sizing with sequence-dependent setups. Int. J. Prod. Res. 45(20), 4873-4894 (2007) 
Almeder, C.: A hybrid optimization approach for multi-level capacitated lot-sizing problems. Eur. J. Oper. Res. 200(2), 599-606 (2010)

Bierwirth, C., Mattfeld, D.: Production scheduling and rescheduling with genetic algorithms. Evolut. Comput. 7(1), 1-18 (1999)

Bierwirth, C., Mattfeld, D.C., Kopfer, H.: On permutation representation for scheduling problems. In: Parallel Problem Solving from Nature, pp. 310-318. Springer, Berlin (1996)

Bitran, G.R., Yanasse, H.H.: Computational complexity of the capacitated lot size problem. Manag. Sci. 28(10), 1174-1186 (1982)

Blum, C., Raidl, G.R.: Hybrid Metaheuristics: Powerful Tools for Optimization. Springer, Berlin (2016)

Branke, J., Mattfeld, D.: Anticipation and flexibility in dynamic scheduling. Int. J. Prod. Res. 43(15), 3103-3129 (2005)

Burke, E.K., Gendreau, M., Hyde, M., Kendall, G., Ochoa, G., Özcan, E., Qu, R.: Hyper-heuristics: a survey of the state of the art. J. Oper. Res. Soc. 64(12), 1695-1724 (2013)

Cheng, R., Gen, M., Tsujimura, Y.: A tutorial survey of job-shop scheduling problems using genetic algorithms-i. Representation. Comput. Ind. Eng. 30(4), 983-997 (1996)

Copil, K., Wörbelauer, M., Meyr, H., Tempelmeier, H.: Simultaneous lotsizing and scheduling problems: a classification and review of models. OR Spectr. 39(1), 1-64 (2017)

Dangelmaier, W., Kaganova, E.: Robust solution approach to CLSP problem with an uncertain demand. In: Robust Manufacturing Control, pp. 455-467. Springer, Berlin (2013)

DiMasi, J.A., Grabowski, H.G.: The cost of biopharmaceutical R\&D: is biotech different? Manag. Decis. Econ. 28(4-5), 469-479 (2007)

Drexl, A., Kimms, A.: Lot sizing and scheduling-survey and extensions. Eur. J. Oper. Res. 99, 221-235 (1997)

Farid, S.S.: Process economics of industrial monoclonal antibody manufacture. J. Chromatogr. B 848(1), 8-18 (2007)

Gatica, G., Papageorgiou, L., Shah, N.: Capacity planning under uncertainty for the pharmaceutical industry. Chem. Eng. Res. Des. 81(6), 665-678 (2003)

Giffler, B., Thompson, G.L.: Algorithms for solving production-scheduling problems. Oper. Res. 8(4), 487-503 (1960)

Goren, H.C., Tunali, S., Jans, R.: A review of applications of genetic algorithms in lot sizing. J. Intell. Manuf. 21(4), 575-590 (2008)

Ho, J.C., Chang, Y.L., Solis, A.O.: Two modifications of the least cost per period heuristic for dynamic lot-sizing. J. Oper. Res. Soc. 57(8), 1005-1013 (2006)

James, R.J.W., Almada-Lobo, B.: Single and parallel machine capacitated lotsizing and scheduling: new iterative MIP-based neighborhood search heuristics. Comput. Oper. Res. 38(12), 1816-1825 (2011)

Jans, R., Degraeve, Z.: Meta-heuristics for dynamic lot sizing: a review and comparison of solution approaches. Eur. J. Oper. Res. 177(3), 1855-1875 (2007)

Jans, R., Degraeve, Z.: Modeling industrial lot sizing problems: a review. Int. J. Prod. Res. 46(6), 1619-1643 (2008)

Jin, Y., Branke, J.: Evolutionary optimization in uncertain evolutionary optimization in uncertain environments-a survey. IEEE Trans. Evolut. Comput. 9(3), 303-317 (2005)

Karimi, B., Fatemi Ghomi, S., Wilson, J.: The capacitated lot sizing problem: a review of models and algorithms. Omega 31(5), 365-378 (2003)

Kimms, A.: A genetic algorithm for multi-level, multi-machine lot sizing and scheduling. Comput. Oper. Res. 26(8), 829-848 (1999)

Laborie, P.: IBM ILOG CP optimizer for detailed scheduling illustrated on three problems. In: van Hoeve WJ, Hooker J (eds) International Conference on Integration of AI and OR Techniques in Constraint Programming for Combinatorial Optimization Problems, Springer, LNCS, vol. 5547, pp. 148-162 (2009)

Lakhdar, K., Zhou, Y., Savery, J., Titchener-Hooker, N.J., Papageorgiou, L.G.: Medium term planning of biopharmaceutical manufacture using mathematical programming. Biotechnol. Prog. 21(5), 14781489 (2005)

Lakhdar, K., Savery, J., Papageorgiou, L.G., Farid, S.S.: Multiobjective long-term planning of biopharmaceutical manufacturing facilities. Biotechnol. Progr. 23(6), 1383-1393 (2007)

Levis, A.A., Papageorgiou, L.G.: A hierarchical solution approach for multi-site capacity planning under uncertainty in the pharmaceutical industry. Comput. Chem. Eng. 28(5), 707-725 (2004) 
Luke, S., Panait, L., Balan, G., Paus, S., Skolicki, Z., Kicinger, R., Popovici, E., Sullivan, K., Harrison, J., Bassett, J., Hubley, R., Desai, A., Chircop, A., Compton, J., Haddon, W., Donnelly, S., Jamil, B., Zelibor, J., Kangas, E., Abidi, F., Mooers, H., O’Beirne, J., Talukder, Khaled Ahsan McDermott J.: ECJ: A Java-based Evolutionary Computation Research System. (2014) https://cs.gmu.edu/ eclab/ projects/ecj/

Mehdizadeh, E., Hajipour, V., Mohammadizadeh, M.R.: A bi-objective multi-item capacitated lot-sizing model: two Pareto-based meta-heuristic algorithms. Int. J. Manag. Sci. Eng. Manag. 11(4), 279-293 (2016)

Özdamar, L., Birbil, S.I.: Hybrid heuristics for the capacitated lot sizing and loading problem with setup times and overtime decisions. Eur. J. Oper. Res. 110(3), 525-547 (1998)

Paul, S., Mytelka, D., Dunwiddie, C., Persinger, C., Munos, B., Lindborg, S., Schacht, A.: How to improve r\&d productivity: the pharmaceutical industry's grand challenge. Nat. Rev. Drug Discov. 9, 203-214 (2010)

Piperagkas, G.S., Konstantaras, I., Skouri, K., Parsopoulos, K.E.: Solving the stochastic dynamic lot-sizing problem through nature-inspired heuristics. Comput. Oper. Res. 39(7), 1555-1565 (2012)

Pitakaso, R., Almeder, C., Doerner, K.F., Hartl, R.F.: A MAX-MIN ant system for unconstrained multi-level lot-sizing problems. Comput. Oper. Res. 34(9), 2533-2552 (2007)

Ramya, R., Rajendran, C., Ziegler, H.: Capacitated lot-sizing problem with production carry-over and set-up splitting: mathematical models. Int. J. Prod. Res. 54(8), 2332-2344 (2016)

Siganporia, C.C., Ghosh, S., Daszkowski, T., Papageorgiou, L.G., Farid, S.S.: Capacity planning for batch and perfusion bioprocesses across multiple biopharmaceutical facilities. Biotechnol. Progr. 30(3), 594-606 (2014)

Silver, E.A., Meal, H.C.: A heuristic for selecting lot size quantities for the case of a deterministic timevarying demand rate and discrete opportunities for replenishment. Prod. Invent. Manag. 14(2), 64-74 (1973)

Wagner, H.M., Whitin, T.M.: Dynamic version of the economic lot size model. Manag. Sci. 5(1), 89-96 (1958)

Walser, J.P., Iyer, R., Venkatasubramanyan, N.: An integer local search method with application to capacitated production planning. In: Proceedings of AAAI-98, pp. 373-379 (1998) 